\title{
Muscular Activity of Lower Extremity Muscles Running on Treadmill Compared with Different Overground Surfaces
}

\author{
Lin Wang ${ }^{1}$, Youlian Hong ${ }^{2}$, Jing Xian $\mathrm{Li}^{3, *}$ \\ ${ }^{1}$ School of Kinesiology, Shanghai University of Sport, Shanghai, China \\ ${ }^{2}$ Department of Sports Medicine, Chengdu Sports University, Chengdu, China \\ ${ }^{3}$ School of Human Kinetics, University of Ottawa, Ottawa, ON K1N 6N5, Canada \\ *Corresponding author: jli@uottawa.ca \\ Received July 04, 2014; Revised July 14, 2014; Accepted July 16, 2014
}

\begin{abstract}
The objective of this study is to compare the muscular activity of lower extremity muscles while running on treadmill and on overground surfaces. A total of 13 experienced heel-to-toe runners participated in the study. Electromyographic (EMG) data of four lower extremity muscles, including rectus femoris, tibialis anterior, biceps femoris, and gastrocnemius, were collected using the Noraxon EMG system while running on a treadmill and on overground surfaces at a running speed of $3.8 \mathrm{~m} / \mathrm{s}$. The obtained data were then analyzed. In this study, throughout the stance phase, the EMG values in the rectus femoris $(P<0.01)$ and the biceps femoris $(P<0.05)$ were higher while running on overground surfaces than those on a treadmill. The EMG values in the rectus femoris $(P<0.05)$ and the biceps femoris $(P<0.05)$ were also higher on concrete than those on grass in the stance phase. Results showed that the muscle activity was significantly different in treadmill running than in overground running. The difference in muscle activity while running on different overground surfaces was also found in this study. Kinematic adjustment of the lower extremity may explain the EMG difference while running on different surfaces.
\end{abstract}

Keywords: running surfaces, muscular activity, Electromyographic, lower extremity, Biomechanics

Cite This Article: Lin Wang, Youlian Hong, and Jing Xian Li, "Muscular Activity of Lower Extremity Muscles Running on Treadmill Compared with Different Overground Surfaces." American Journal of Sports Science and Medicine, vol. 2, no. 4 (2014): 161-165. doi: 10.12691/ajssm-2-4-8.

\section{Introduction}

Running is one of the most popular sports activities. People run on different surfaces. Surfaces for overground running include concrete, asphalt, sports track made from synthetic rubber, and natural turf $[1,2]$. Meanwhile, treadmills are widely used in laboratory settings for training and research that require control on speed and slope [3].

The increasing use of treadmills has forwarded questions on the difference in biomechanics characteristics between running on a treadmill and on overground surfaces. To date, perspectives on whether the treadmillbased analysis of running mechanics can simulate overground running mechanics remain contradictory $[4,5]$. Published studies mainly focused on running kinematics and kinetics. Contradictory results are likewise shown in the kinematic analysis. Wank et al. found that compared with running on overground surfaces, treadmill running exhibits a shorter flight phase, decreased stride length, and increased cadence at a moderate speed ranging from 3.3 $\mathrm{m} / \mathrm{s}$ to $4.8 \mathrm{~m} / \mathrm{s}$ [6]. Other studies found that some kinematic variables (e.g., hip adduction angle, hip internal/external rotation, ankle eversion, and maximal pelvic rotation) of the treadmill gait are slightly different from those of the overground gait [5,7]. In the kinematic analysis of a study, no significant difference was found in vertical ground reaction force between treadmill and overground running at a constant running speed [7]. In addition, several studies observed an in-shod plantar pressure during treadmill and overground running $[8,9]$. These studies found that compared with overground running, treadmill running has a lower magnitude of maximum plantar pressure at the plantar area. Kinematic changes in the ankle joint complex during treadmill running attribute the difference in the plantar pressure $[8,9]$. Furthermore, the manifestation of biomechanics changes in treadmill running in the changes in neuromuscular activation is still under debate $[6,8]$.

When running on different surfaces, runners adapt their lower extremity kinematics and stiffness to maintain similar impact forces [10,11]. In previous studies, researchers found that kinematic adaptation is associated with neuromuscular adaptation while running on different surfaces $[8,12]$. A few studies attempted to identify the differences in muscular activity while running on different surfaces $[6,8]$. In these studies, electromyography (EMG) was used to measure muscular activation during running. In several earlier studies, researchers failed to identify the differences in amplitudes and coordination of EMGrelated parameters between treadmill and overground 
running [13,14]. Wank et al. observed similar EMG patterns of the leg muscles in comparing overground and treadmill running at speeds of 4 and $6 \mathrm{~m} / \mathrm{s}$ [6]. In the same study, researchers reported that the biceps femoris showed higher magnitude and longer activity duration at ground contact and swing phase during treadmill running than other muscles. The vastus lateralis also showed lower amplitudes at ground contact. Baur et al. found that during overground running, EMG exhibited a later onset, a later maximum, and a shorter total time in the peroneus longus than that in treadmill running, while the soleus showed higher amplitude during overground running at the pushoff phase [8].

Despite the difference in muscular activity findings between treadmill and overground running in previous studies, the types of overground surfaces were not described. The hardness of overground surfaces affects the muscular activity of the runner [12]. To date, no investigation has been conducted on the differences in EMG parameters when runners run on different overground surfaces and on the treadmill. Thus, the present study aims to examine the differences in muscle activities when running on different overground surfaces and on the treadmill. The results of this study will demonstrate advanced differences in muscular activation while running on a treadmill and on different overground surfaces, which will determine if treadmill running can be used to simulate the muscle activity of overground running.

\section{Methods}

\subsection{Subjects}

Thirteen young male students (aged $22.4 \pm 3.9$ years, body mass of $63.6 \pm 9.2 \mathrm{~kg}$, and body height of $170.6 \pm$ $6.2 \mathrm{~cm}$ ) volunteered to participate in the study. All participants were right-leg dominant, heel strikers in running, and had a shoe size of 41 (European standard). The participants were experienced treadmill or overground surface runners and ran at least $20 \mathrm{~km}$ per week. Only male participants were recruited to eliminate gender differences in the running biomechanics. The participants had no history of diseases associated with the neuromuscular system and suffered no sports injuries in the last six months prior to the study. Prior to the experiments, the participants were provided an informed consent. The study was approved by the Ethics Committee of the local university.

\subsection{Running Surfaces and Running Shoes}

Concrete and asphalt are the most commonly used surfaces for recreational and marathon runs. Natural grass surfaces had been previously examined in the study of plantar loads while running and performing specific sports movements. In the present study, three overground surfaces, namely, concrete (C), synthetic rubber (R), and natural grass $(\mathrm{G})$ were studied. Natural grass and rubber surfaces comprise the standard natural grass soccer field and the standard synthetic rubber running track, respectively. Treadmill running tests were conducted on a treadmill (T) (6300HR, SportsArt Fitness, USA).

A pair of new running shoes with European size of 41 (TN600-neutral, ASICS, Japan) was assigned to each participant. The running tests were performed on each surface using the said footwear.

\subsection{Testing Protocol}

During the running trials, the EMG signals were acquired and transmitted by the Noraxon TeleMyo (Noraxon USA Inc., Scottsdale, USA) telemetered EMG system (bandwidth from $10 \mathrm{~Hz}$ to $350 \mathrm{~Hz}$ ). The frequency of the EMG data acquisition was set at $1000 \mathrm{~Hz}$. The EMG collection was synchronized with the video data recording using the Ariel Performance Analysis System (Ariel Dynamics Inc., Trabuco Canyon, USA). The EMG data were collected from four lower extremity muscles, namely, rectus femoris, tibialis anterior, biceps femoris, and gastrocnemius [12]. Before the electrode placement, the participant's skin was shaved and cleansed with alcohol. Bipolar surface electrodes (Noraxon Dual \#272, US) were attached to the participant's skin at the midline of the muscle belly [15]. To reduce inconsistency and inter-subject variability in normalizing the EMG signal [16], the EMG signal was normalized to a reference activity rather than to a maximum voluntary contraction. Four controlled reference postures, namely, squatting, lower leg raised to $90^{\circ}$, dorsiflexion, and plantar flexion were implemented to normalize the muscles under study [17]. The EMG signals in the selected postures were recorded under submaximal isometric contraction.

The treadmill running test was conducted in an indoor laboratory. Each participant ran six minutes on a treadmill at $3.3 \mathrm{~m} / \mathrm{s}$ for warm-up [18]. Subsequently, they were instructed to run on the treadmill at a velocity of $3.8 \mathrm{~m} / \mathrm{s}$ for $2 \mathrm{~min}$ for data collection. Five successful steps of the right-foot stance phase during the last minute were measured for data analysis.

The overground running test was conducted on a $30 \mathrm{~m}$ straight runway. The first $15 \mathrm{~m}$ of the runway was the acceleration zone, followed by $5 \mathrm{~m}(15 \mathrm{~m}$ to $20 \mathrm{~m})$ of the measurement zone where participants ran at a velocity of $3.8 \mathrm{~m} / \mathrm{s}$. This velocity was consistent with that employed in previous studies $[1,19]$. The velocity was timed using an infrared timing system (Brower Timing System, USA). The timers were placed at the start and end points of the measurement zone. Each participant ran for $6 \mathrm{~min}$ on a standard running track at his preferred velocity to warm up. After warm up and prior to data collection, each participant was allowed as many practice trials as necessary to achieve a smooth running pattern, with controlled velocity of $3.8 \mathrm{~m} / \mathrm{s}$. The trial was accepted when the running velocity was within $5 \%$ of the controlled velocity on the $5 \mathrm{~m}$ measurement zone. On each running surface, participants completed five successful trials. In each successful trial, plantar load data of at least one complete right-foot stance were collected. The right-foot stance indicated the phase from heel strike to toe push off of the right foot during running. Five steps on each surface were used in the data reduction. The order of running surfaces was randomly assigned to each participant. The same protocol was used in our previous study [9].

\subsection{Data Reduction and Analysis}

All EMG raw data were processed by the Noraxon EMG system. The raw EMG signal was filtered using the band-pass filter with bandwidth ranging from $20 \mathrm{~Hz}$ to 
$500 \mathrm{~Hz}$, and then the signal was full wave-rectified. By selecting a complete stride, the magnitude of the signal recorded from each of the channels was normalized to the maximum magnitude obtained from the submaximal isometric contraction tests. The time normalization of the stance and the swing phases was separately performed for each of the running trials. Each cycle was divided into four phases (Montgomery III, Pink, and Perry, 1994). By definition, one stride or cycle is the period from the initial contact of one foot to the initial contact of the same foot. A complete running stride is considered as two steps. Each step is defined as the initial contact of one foot and then the initial contact of the contralateral foot. The foot experiences the support and the swing phases [20]: the stance (from the right-heel touchdown to the right toe off),

the early swing (from the right toe off to the left-heel touchdown), the middle swing (from the left-heel touchdown to the left toe off), and the late swing (from the left toe off to the right-heel touchdown).

All data are presented as mean (standard deviation, SD). The comparison of surfaces was performed using ANOVA for repeated measurement analysis on selected EMG variables. Significance was at alpha $<0.05$, and Bonferroni adjustment was used to correct multiple measurements. The $95 \%$ confidence intervals (CI) for the mean difference in each variable among the four surfaces were calculated to determine the range of differences.

\section{Results}

Table 1. Mean (SD) of muscle activity parameters (magnitude normalized in ratio)

\begin{tabular}{|c|c|c|c|c|c|}
\hline Muscle \& running phase & $\mathrm{T}$ & $\mathrm{R}$ & $\mathrm{G}$ & $\mathrm{C}$ & \\
\hline Rectus Femoris Phase 1 & $0.037(0.023)$ & $0.213(0.076)$ & $0.154(0.045)$ & $0.247(0.130)$ & $*, \#, \S, \dagger$ \\
\hline Rectus Femoris Phase 2 & $0.038(0.022)$ & $0.070(0.021)$ & $0.042(0.016)$ & $0.091(0.039)$ & \\
\hline Rectus Femoris Phase 3 & $0.037(0.019)$ & $0.037(0.020)$ & $0.030(0.021)$ & $0.051(0.052)$ & \\
\hline Rectus Femoris Phase 4 & $0.024(0.005)$ & $0.017(0.008)$ & $0.030(0.006)$ & $0.050(0.009)$ & \\
\hline Tibialis Anterior Phase 1 & $0.083(0.031)$ & $0.105(0.042)$ & $0.114(0.061)$ & $0.144(0.060)$ & \\
\hline Tibialis Anterior Phase 2 & $0.066(0.011)$ & $0.079(0.024)$ & $0.093(0.006)$ & $0.140(0.004)$ & \\
\hline Tibialis Anterior Phase 3 & $0.092(0.022)$ & $0.102(0.035)$ & $0.100(0.032)$ & $0.129(0.022)$ & \\
\hline Tibialis Anterior Phase 4 & $0.122(0.095)$ & $0.113(0.042)$ & $0.164(0.140)$ & $0.139(0.105)$ & \\
\hline Biceps Femoris Phase 1 & $0.048(0.028)$ & $0.133(0.072)$ & $0.099(0.062)$ & $0.128(0.126)$ & $*, \#, \S, \dagger$ \\
\hline Biceps Femoris Phase 2 & $0.024(0.012)$ & $0.045(0.011)$ & $0.057(0.011)$ & $0.083(0.025)$ & \\
\hline Biceps Femoris Phase 3 & $0.064(0.053)$ & $0.098(0.070)$ & $0.102(0.070)$ & $0.133(0.086)$ & \\
\hline Biceps Femoris Phase 4 & $0.124(0.107)$ & $0.160(0.138)$ & $0.102(0.068)$ & $0.128(0.080)$ & \\
\hline Gastrocnemius Phase 1 & $0.474(0.311)$ & $0.622(0.230)$ & $0.609(0.399)$ & $0.600(0.405)$ & \\
\hline Gastrocnemius Phase 2 & $0.144(0.031)$ & $0.236(0.057)$ & $0.174(0.073)$ & $0.179(0.045)$ & \\
\hline Gastrocnemius Phase 3 & $0.070(0.043)$ & $0.090(0.078)$ & $0.052(0.050)$ & $0.074(0.054)$ & \\
\hline Gastrocnemius Phase 4 & $0.066(0.018)$ & $0.078(0.004)$ & $0.128(0.007)$ & $0.179(0.008)$ & \\
\hline
\end{tabular}

Note: T,Treadmill; R, Synthetic rubber; G,Grass; C,Concrete

Phase 1,Stance phase; Phase 2,Early swing; Phase 3,Middle swing; Phase 4,Late swing

$*, P<0.05$, T vs. Ta; \#, $P<0.05$, T vs. G; $\S, P<0.05$, T vs.C; $\dagger, P<0.05$, G vs.C;
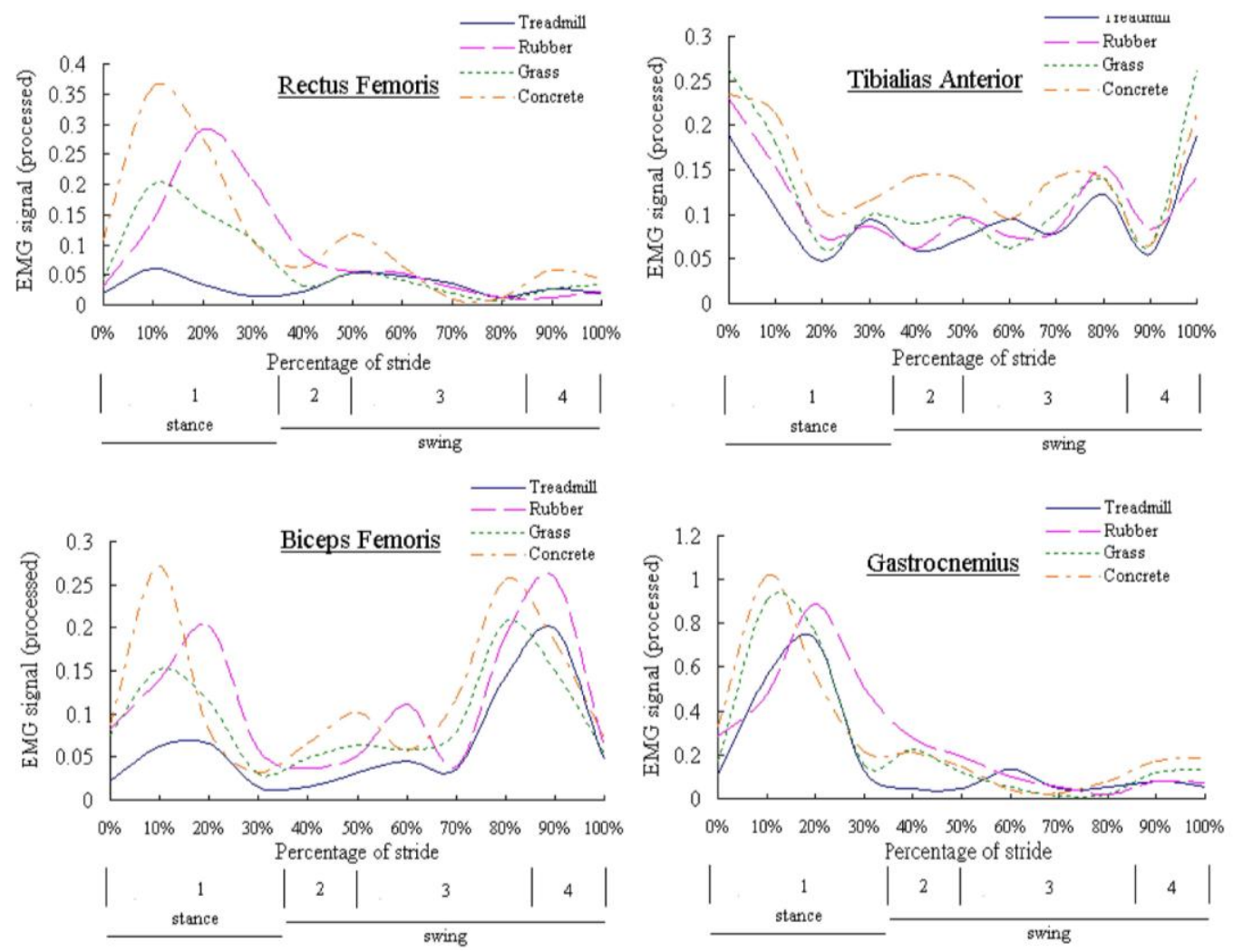

Figure 1. The EMG profile of four muscle groups of one stride 
In the study, different EMG patterns between treadmill and overground running were found (Figure 1). Significant differences were observed in the stance phase in the rectus femoris and the biceps femoris. Throughout the stance phase, the EMG values in the rectus femoris $(P<0.01,95 \% \mathrm{CI}$ for mean difference, $\mathrm{R}: \mathrm{T}=0.273$ to $0.079, \mathrm{G}: \mathrm{T}=0.183$ to $0.050, \mathrm{C}: \mathrm{T}=0.360$ to 0.060$)$ and the biceps femoris $(P<0.05,95 \% \mathrm{CI}$ for mean difference, $\mathrm{R}: \mathrm{T}=0.183$ to $0.010, \mathrm{G}: \mathrm{T}=0.139$ to $0.030, \mathrm{C}: \mathrm{T}=0.231$ to 0.070 ) were higher on overground surfaces than those on the treadmill. Furthermore, the EMG values in the rectus femoris $(P<0.05,95 \% \mathrm{CI}$ for mean difference $=$ 0.179 to 0.007$)$ and the biceps femoris $(P<0.05,95 \% \mathrm{CI}$ for mean difference $=0.121$ to 0.006 ) were higher on concrete than those on grass. No significant differences were found for all muscles in the swing phases (Table 1).

\section{Discussion}

In this study, the primary finding was that the muscle activity of the rectus femoris and the biceps femoris has a lower magnitude of EMG values in treadmill running than that in overground running during the stance phase. The EMG values in treadmill and overground running showed similar activity patterns during the swing phase.

The result on the rectus femoris was consistent with that by Wank et al. [6]. Wank et al. found a higher EMG magnitude of the biceps femoris during the last part of the ground contact in treadmill running than that in overground running [6]. This result is in contrast to the findings in the present study in which a lower EMG magnitude of the muscle during the stance phase on the treadmill was found than that on overground surfaces. The difference in running speed ( 4 and $6 \mathrm{~m} / \mathrm{s}$ in Wank et al.'s study $v s .3 .8 \mathrm{~m} / \mathrm{s}$ in this study) and the division of running gait phases (three phases in Wank et al.'s study vs. four phases in this study) may also contribute to the varied results between the two studies. In treadmill running, the body is not necessarily pushed forward continuously. Thus, not much energy is needed to provide the forward movement of the body's center of gravity (CG) compared with that in overground running during the heel touchdown to the toe-off period. This explanation can be supported by the kinematic findings [21]. In the stance phase, significant differences were observed on the parameters of the trunk angle between treadmill and overground running. Treadmill running showed less forward lean of the trunk. As mentioned earlier, this difference is because, compared with overground running, no forward movement of the trunk was necessary in treadmill running and the running speed was maintained by the treadmill belt. Novacheck proposed that CG can be moved in front of the support foot in the stance phase by a greater forward trunk lean, while a greater horizontal GRF can be exerted on the contact surface [22]. Therefore, in treadmill running, $\mathrm{CG}$ of the runner does not move forward and less horizontal GRF is needed. This kinematic characteristic can be reflected by the observation in the muscle activity. The less horizontal GRF necessary in treadmill running, the lower is the magnitude of muscle activity of the rectus femoris and the biceps femoris in treadmill running than that in overground running during the stance phase.
Moreover, in the stance phase, the muscle activity of the rectus femoris and the biceps femoris showed lower magnitude in grass running than that in concrete running. The differences in muscle activity levels may be associated with the stiffness of the running surfaces. Previous studies showed that the hard surface with high stiffness level led to the increase in the touchdown impact force [18,23]. Consequently, a higher force was transmitted to the leg, and a greater contraction was required to provide the support. In a recent study, similar maximal plantar forces were found while running on different overground surfaces at total foot and different plantar areas [24]. Several studies found that increased surface hardness induces kinematic changes in the lower extremity on the sagittal plane $[10,18]$. Lower extremity kinematics and stiffness adaptations to different overground running surfaces have been interpreted as a form of active adaptation in maintaining similar impact forces $[10,11,18]$. These adaptations included larger ankle and knee flexion [10] and larger knee and hip flexion at heel strike on more rigid surfaces [11]. The runner can adapt kinematic characteristics by adjusting the musculoskeletal system while running on different surfaces to maintain similar impact force $[10,11,18]$. The findings in the present study may provide advanced evidence on the muscular adjustment of the lower extremity when a runner runs on different overground surfaces.

Overall, significant differences were found in muscular activities between treadmill and overground running. Therefore, treadmill running may be considered as a different movement task that requires a specific muscle action. Treadmill running may also be proposed as an effective method for athletic training or physiological testing in laboratories because of its EMG characteristics in specific muscles. However, researchers should be cautious in applying the results from the treadmill test. The results obtained from the current trend of shoe testing on the treadmill may not accurately reveal the real functional response of the shoes when used in overground running. Moreover, the test results showed that substantial changes in the lower extremity muscle activity occur in response to the altered surface during running. Changing the hardness of the surface can alter the activity of the lower extremity muscle. By selecting different surfaces for training purposes, different training effects can be achieved.

\section{Conclusion}

The results showed that muscle activity is significantly different in treadmill running than that in overground running. Moreover, a difference in muscle activity while running on different surfaces was found. The kinematic adjustment of the lower extremity may explain the EMG difference when running on different surfaces.

\section{Competing Interests}

The authors declare that they have no competing interests. 


\section{References}

[1] Ford, K. R., Manson, N. A., Evans, B. J., Myer, G. D., Gwin, R. C., Heidt, R. S. Jr., Hewett, T. E., "Comparison of in-shoe foot loading patterns on natural grass and synthetic turf." Journal of Science and Medicine in Sport, 9 (6), 433-440. 2006.

[2] Tessutti, V., Trombini-Souza, F., Ribeiro, A. P., Nunes, A. L., Sacco-Ide, C., "In-shoe plantar pressure distribution during running on natural grass and asphalt in recreational runners". Journal of Science and Medicine in Sport, 13 (1), 151-155. 2010.

[3] Lavcanska, V., Taylor, N.F., Schache, A.G., "Familiarization to treadmill running in young unimpaired adults". Human Movement Science, 24 (4), 544-557. 2005.

[4] Kram, R., Griffin, T.M., Donelan, J.M., Chang, Y. H., "Force treadmill for measuring vertical and horizontal ground reaction forces". Journal of Applied Physiology, 85 (2), 764-769. 1998.

[5] Schache, A.G., Blanch, P.D., Rath, D.A., Wrigley, T.V., Starr, R., Bennell, K.L., "A comparison of overground and treadmil running for measuring the three-dimensional kinematics of the lumbo-pelvic-hip complex". Clinical Biomechanics, 16 (8), $667-$ 680. 2001.

[6] Wank, V., Frick, U., Schmidtbleicher, D., "Kinematics and electromyography of lower limb muscles in overground an treadmill running". International Journal of Sports Medicine, 19 (7), 455-461.1998.

[7] Riley, P.O., Dicharry, J., Franz, J., Della Croce, U., Wilder, R.P. Kerrigan, D.C., "A kinematics and kinetic comparison of overground and treadmill running". Medicine and Science in Sports and Exercise, 40 (6), 1093-1100. 2008.

[8] Baur, H., Hirschmüller, A., Müller, S., Gollhofer, A., Mayer, F, "Muscular activity in treadmill and overground running". Isokinetics and Exercise Science, 15 (2), 166-171. 2007.

[9] Hong, Y., Wang, L., Li, J.X. \& Zhou, J.H. "Comparison of plantar loads during treadmill and overground running". Journal of Science and Medicine in Sport, 15 (6), 554-560. 2012.

[10] Dixon, S.J., Collop, A.C., Batt, M.E., "Surface effects on ground reaction forces and lower extremity kinematics in running". Medicine and Science in Sports and Exercise, 32 (11), 1919-1926. 2000.

[11] Ferris, D.P., Louie, M., Farley, C.T., "Running in the real world: adjusting leg stiffness for different surfaces". Proceedings Biological Sciences, 265(1400), 989-994. 1998.
[12] Pinnington, H.C., Lloyd, D.G., Besier, T.F., Dawson, B., "Kinematic and electromyography analysis of submaximal differences running on a firm surface compared with soft, dry sand". European Journal of Applied Physiology, 94 (3), 242-253. 2005.

[13] Arsenault, A.B., Winter, D.A., Marteniuk, R.G., "Treadmill versus walkway locomotion in humans: an EMG study". Ergonomics, 29 (5), 665-676. 1986.

[14] Schwab, G.H., Moynes, D.R., Jobe, F.W., Perry, J., "Lower extremity electromyographic analysis of running gait". Clinical Orthopaedics \& Related Research, 176, 166-170. 1983.

[15] De Luca, C.J., "The use of surface electromyography in biomechanics". Journal of Applied Biomechanics, 13 (2), 135-163. 1997.

[16] Lehman, G.J., McGill, S.M., "The importance of normalization in the interpretation of surface electromyography: A proof of principle". Journal of Manipulative and Physiological Therapeutics, 22 (7), 444-446. 1999.

[17] Fong, D.T.P., Hong, Y., Li, J.X., "Lower extremity preventive measures to slips-joint moments and myoeletric analysis". Ergonomics, 51 (12), 1830-1846. 2008.

[18] Hardin, E.C., van den Bogert, A.J., Hamill, J., "Kinematic adaptations during running: effects of footwear, surface, and duration". Medicine and Science in Sports and Exercise, 36 (5), 838-844. 2004.

[19] Tillman, M.D., Fiolkowski, P., Bauer, J.A., Reisinger, K.D., "Inshoe plantar measurements during running on different surfaces: changes in temporal and kinetic parameters". Sports Engineering, 5 (3), 121-128. 2002.

[20] Birrer, R.B., Buzermanis, S., DellaCorte, M.P., Grisalfi, P.J., "Biomechanics of Running". in Textbook of Running Medicine, McGraw-Hill, Medical Publishing Division, 2001, 11-19.

[21] Mok, K.M., Lee, J., Chung, M., Hong, Y. "A kinematic comparison of running on treadmill and overground surfaces". in 27th International Conference on Biomechanics in Sports, International Society of Biomechanics, 1-4.

[22] Novacheck, T.F., "The biomechanics of running". Gait and Posture, 7 (1), 77-95. 1998.

[23] Bulter, J.R., Crowell, III. H.P., Davis, I.M., (2003). "Lower extremities stiffness: Implications for performance and injury". Clinical Biomechanics, 18 (6), 511-517. 2003.

[24] Wang, L., Hong, Y., Li, J.X., Zhou, J.H., "Comparison on plantar load during running on different overground surfaces". Research in Sports Medicine, 20 (2), 75-78. 2012. 